\title{
CLASSIFICATION OF SPACES OF THE SAME $n$-TYPE FOR ALL $n$
}

\author{
CLARENCE WILKERSON
}

\begin{abstract}
The set of homotopy equivalence classes of $\mathrm{CW}$-spaces $Y$ for which the Postnikov approximations are homotopy equivalent to those of a given space $X$ is shown to be in one-to-one correspondence with $\lim ^{1}$ of a tower of homotopy automorphism groups.
\end{abstract}

Recall that two CW-spaces $X$ and $Y$ are said to have the same $n$-type if the $n$th Postnikov approximations $X^{(n)}$ and $Y^{(n)}$ are homotopy equivalent. The homotopy classification problem for infinite dimensional complexes can be studied in two steps. First, classify all finite dimensional complexes (!) and then classify for a given $X$ all complexes of the same $n$-type for all $n$ as $X$. The purpose of this note is to codify this second step.

Theorem I. Let $X$ be a connected $C W$-space. Denote by $S N T(X)$ the set of homotopy equivalence classes of $C W$-spaces $Y$ such that $Y$ has the same n-type for all $n$ as $X$. Then $S N T(X)$ is in one-to-one correspondence with the pointed set $\lim ^{1}\left(\operatorname{Aut}\left(X^{(n)}\right)\right)$, where $\operatorname{Aut}\left(X^{(n)}\right)$ is the group of homotopy classes of homotopy equivalences of $X^{(n)}$.

The relevance of $\lim ^{1}$-type arguments is clear from the work of Adams [1] and Gray [4], but Theorem I seems to be the first explicit formulation of the classification problem. The first application is to study some cases for which $\operatorname{SNT}(X)=\{X\}$.

Corollary II. Let $X$ be a simply connected $C W$-space such that $\pi_{i}(X)$ is finitely generated in each dimension.

(a) (Adams [1]) If $\pi_{i}(X)$ is finite for all $i>0$, then $S N T(X)=\{X\}$.

(b) If $X_{0}$ denotes the rationalization of $X$ [8], [3], [5], then $\operatorname{SNT}\left(X_{0}\right)=\left\{X_{0}\right\}$.

(c) If $\hat{X}$ denotes the profinite completion of $X[8]$, then $S N T(\hat{X})=\{\hat{X}\}$.

The proof of the corollary is the demonstration that for the towers of automorphism groups involved, the $\lim ^{1}$ set reduces to the one point set.

Proposition III. Let $X$ be a simply connected $C W$-space such that $\pi_{i}(X)$ is finitely generated in each dimension. Then $\lim ^{1}\left(\operatorname{Aut}\left(X^{(n)}\right)\right) \approx \lim ^{1}(\operatorname{Im}(n))$, where $\operatorname{Im}(n)$ denotes $\left\{\operatorname{image}\left(\operatorname{Aut}\left(X^{(n)}\right) \rightarrow \operatorname{Aut}\left(X_{0}^{((n)}\right)\right)\right\}$

Finally, we study in some detail an example of a rational $H$-space $X$ such that $S N T\left(X_{p}\right) \neq\left\{X_{p}\right\}$ for any prime $p$. This example is motivated by Gray's proof [4] that for $W=\mathbf{C} P^{\infty} \times \Omega S^{3}, S N T(W) \neq\{W\}$. Our example shows

Received by the editors September 29, 1975.

AMS (MOS) subject classifications (1970). Primary 55G45, 55D10. 
that the finite dimensionality hypothesis of Theorem 1.1 of [2] is necessary: there the result is that if $Y^{\prime}$ and $Y^{\prime \prime}$ are simply connected finite dimensional rational $H$-spaces such that $\hat{Y}_{p}^{\prime} \simeq \hat{Y}_{p}^{\prime \prime}$, then $Y_{p}^{\prime} \simeq Y_{p}^{\prime \prime}$. However, Corollary II(c) and the example above show that this cannot hold in general.

We note that the classification argument of $\S I$ is a variant of the standard "principal homogeneous space" argument of algebraic geometry, and can be modified to classify the possible pullbacks of other diagrams of spaces in terms of the automorphism groups, e.g., $\$ 3$ of Wilkerson [10]. I would like to acknowledge helpful discussions with J. F. Adams, A. K. Bousfield, J. Cohen, G. Porter and P. Trauber on this material.

I. The classification. We first recall the definition of the $\lim ^{1}$ functor for a tower of groups $\left(G_{n}, q_{n}\right)$, where $q_{n}: G_{n} \rightarrow G_{n-1}:$ there is an action of the product group $\Pi_{n} G_{n}$ on the product set $\Pi_{n} G_{n}$, defined as $\left(\gamma_{n}\right)\left(\alpha_{n}\right)$ $=\left(\gamma_{n} \alpha_{n}\left(q_{n+1}\left(\gamma_{n+1}\right)^{-1}\right)\right)$. The orbit set of this action is denoted as $\lim ^{1} G_{n}$ and has as a base point the equivalence class of $\left(e_{n}\right)$, where $e_{n}$ is the identity element of $G_{n}$.

For the proof of Theorem I, the simplicial setting offers some technical advantages. The Moore-Postnikov decomposition is functorial and inverse limits can be taken as inverse limits of sets. $X$ is to be considered a pointed connected minimal Kan simplicial set in the following. We first recall the Moore-Postnikov decomposition of $X$, see, for example, May [6]. Define an equivalence relation on the simplices of $X, x \sim^{n} y$, if and only if all corresponding faces of $x$ and $y$ of dimension less than or equal to $n$ agree. $X^{(n)}$, the quotient of $X$ by $\sim^{n}$, is again a pointed connected minimal Kan complex. $X \rightarrow X^{(n)}$ is a Kan fibration, and the triangle

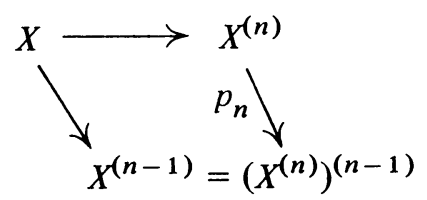

commutes. If $\lim _{\leftarrow}$ is applied degree-wise to the tower of fibrations $\left(X^{(n)}, p_{n}\right)$, then $X=\lim \left(\overleftarrow{X}^{(n)}, p_{n}\right)$ as simplicial sets. It is also clear from the construction that any simplicial map $f: X \rightarrow Y$ induces $f_{n}: X^{(n)} \rightarrow Y^{(n)}$ for all $n$, which commutes with the projections, and conversely, a family of maps $f_{n}$ which commutes with the projections determines a unique map $f: X \rightarrow Y$.

Note that if $X$ and $Y$ are minimal complexes, then any homotopy equivalence $f: X \rightarrow Y$ is actually a simplicial isomorphism. This will be convenient for technical reasons. Define $\operatorname{Simp} \operatorname{Aut}\left(X^{(n)}\right)$ to be the set of all simplicial isomorphisms of $X^{(n)}$ to itself. We begin the classification of $S N T(X)$ by defining a set map $\varphi: \Pi_{n} \operatorname{Simp} \operatorname{Aut}\left(X^{(n)}\right) \rightarrow \operatorname{SNT}(X)$. Let $\bar{\alpha}$ $=\left(\alpha_{0}, \alpha_{1}, \ldots\right)$ belong to $\Pi_{n} \operatorname{Simp} \operatorname{Aut}\left(X^{(n)}\right)$. Define

$$
\varphi(\bar{\alpha})=X_{\bar{\alpha}}=\lim _{\leftarrow}\left(X^{(n)}, \alpha_{n-1} p_{n}\right),
$$

where the inverse limit is applied degree-wise. Then $X_{\bar{\alpha}}$ is again a minimal Kan complex and belongs to $S N T(X)$. The homotopy lifting property of the fibrations $\alpha_{n-1} p_{n}: X^{(n)} \rightarrow X^{(n-1)}$ shows that if $\alpha_{n}$ is homotopic to $\alpha_{n}^{\prime}$ for all $n$, 
then $X_{\bar{\alpha}}^{\prime}$ is homotopy equivalent to $X_{\bar{\alpha}}$. Thus $\varphi: \Pi_{n} \operatorname{Aut}\left(X^{(n)}\right) \rightarrow \operatorname{SNT}(X)$ is well defined.

We show now that $\varphi$ is onto. Let $Y$ be a minimal Kan complex in $S N T(X)$. Choose for each $n$ a homotopy equivalence (simplicial isomorphism) $g_{n}$ : $Y^{(n)} \rightarrow X^{(n)}$. Denote the map induced by $g_{n+1}$ as $\left(g_{n+1}\right)_{n}: Y^{(n)} \rightarrow X^{(n)}$ and define $\left(\alpha_{n}\right)=\left(\left(g_{n}\right)\left(g_{n+1}\right)_{n}^{-1}\right)$. Then $Y=X_{\bar{\alpha}}=\lim \left(X_{n}, \alpha_{n-1} p_{n}\right)$ as simplicial sets. That is, $\varphi$ is surjective.

We now show that $\varphi$ remains well defined after passage to the quotient set $\lim ^{1}\left(\operatorname{Aut}\left(X^{(n)}\right)\right)$; that is, if $\bar{\alpha}=\bar{\gamma} \circ \bar{\beta}$, then $X_{\bar{\alpha}} \simeq X_{\bar{\beta}}$. The square below commutes up to homotopy:

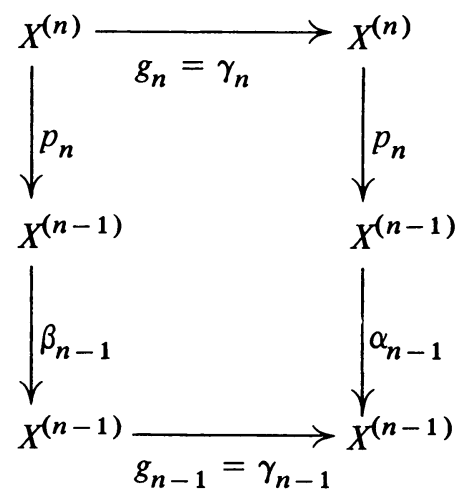

We wish to find a map homotopic to $\gamma_{n}$ such that the diagram commutes. Assume this has been done at all levels below $n$, and that the $\gamma_{m}=g_{m}$ in the diagram are the modified maps, for $m<n$. Then the covering homotopy property of the fibrations shows that $g_{n}$ homotopic to the original $\gamma_{n}$ exists such that the diagram commutes. Thus, there is an isomorphism $g: X_{\bar{\beta}} \rightarrow X_{\bar{\alpha}}$. Conversely, given an isomorphism $g: X_{\beta} \rightarrow X_{\bar{\alpha}}$, define $\gamma_{n}=g_{n}$ and it is easy to see that $\bar{\alpha}=\bar{\gamma} \circ \bar{\beta}$. Thus $\varphi: \lim ^{1}\left(\operatorname{Aut}\left(X^{(n)}\right)\right) \rightarrow S N T(X)$ is monic. That is $\varphi$ is an isomorphism of pointed sets, since it takes the equivalence class of $\left(e_{n}\right)$ to $\{X\}$, and is monic and surjective.

The proof for the case that $X^{\prime}$ is a $\mathrm{CW}$-complex now follows by taking the $X$ above to be a minimal subcomplex of the singular complex of $X^{\prime}$ and observing that the geometric realization of $X^{(n)}$ gives a Postnikov tower for $X^{\prime}$, up to homotopy.

II. Vanishing of $\lim ^{1}$. Recall that a tower of groups $\left(G_{n}, q_{n}\right)$ is Mittag-Leffler if for each $n$, there exists $N_{n}$ such that image $q_{n+m} \ldots q_{n+1}$ = image $q_{n+N_{n}} \ldots q_{n+1}$ for all $m>N_{n}$. If $\left(G_{n}, q_{n}\right)$ is Mittag-Leffler, then $\lim ^{1} G_{n}=*$; see Bousfield and Kan [3]. Therefore Corollary II(b) follows directly from Lemma 2.1.

Lemma 2.1. If $X$ is a simply connected $C W$-space such that $\pi_{i}(X) \otimes \mathbf{Q}$ is finitely generated over $\mathbf{Q}$ for each $i$, then the tower of $\operatorname{groups}\left(\operatorname{Aut}\left(X_{0}^{(n)}\right), q_{n}\right)$ is Mittag-Leffler.

Proof. By Wilkerson [10] or Sullivan [9], there is a finitely generated Q-Hopf-algebra $S(n)$ such that $\operatorname{Aut}\left(X_{0}^{(n)}\right)=\operatorname{Hom}_{\mathbf{Q} \text {-alg }}[S(n), \mathbf{Q}]$ and $p_{n}: X_{0}^{(n)}$ $\rightarrow X_{0}^{(n-1)}$ induces $\tilde{p}_{n}: S(n-1) \rightarrow S(n)$ a map of $\mathbf{Q}$-algebras. That is, 
$\operatorname{Aut}\left(X_{0}^{(n)}\right)$ is a linear algebraic group over $\mathbf{Q}$, and $q_{n}$ is a morphism of linear algebraic groups. Thus the image of each $\operatorname{Aut}\left(X_{0}^{(n+k)}\right)$ is a closed subgroup of $\operatorname{Aut}\left(X_{0}^{(n)}\right)$ in the Zariski topology. That is, there is an ideal $I_{k, n}$ of $S(n)$ such that the image is exactly the elements of $\operatorname{Hom}_{\mathbf{Q} \text {-alg }}[S(n), Q]$ which annihilate $I_{k, n}$. Since $I_{k, n}$ is properly contained in $I_{k+1, n}$ if the image subgroups do not coincide, the noetherian property of $S(n)$ shows that $\operatorname{Aut}\left(X_{0}^{(n)}\right)$ is MittagLeffler.

LEMMA 2.2. If $\left(G_{n}, q_{n}\right)$ is a tower of compact topological groups and continuous homomorphisms, then $\lim ^{1} G_{n}=*$.

Proof. This is an easy application of the finite intersection characterization of compactness and is well known.

LeMma 2.3. Let $X$ be a simply connected $C W$-complex such that $\pi_{*}(X)$ is finitely generated over some subring of $\mathbf{Q}$. Then $\operatorname{Aut}\left(\hat{X}_{p}^{(n)}\right)$ is compact and $q_{n}: \operatorname{Aut}\left(\hat{X}_{p}^{(n)}\right) \rightarrow \operatorname{Aut}\left(\hat{X}_{p}^{(n-1)}\right)$ is continuous.

Proof. This is similar to arguments in Sullivan [8]. By Bousfield and Kan [3], $\hat{X}_{p}^{(n)} \simeq\left(X^{(n)}\right)_{p}^{\hat{n}} \simeq R_{\infty} X^{(n)}$ for $R=\mathbf{Z} / p \mathbf{Z} . R_{\infty} X^{(n)}$ is the inverse limit of a functorial tower of fibrations $R_{s} X^{(n)}$, where for $R=\mathbf{Z} / p \mathbf{Z}$, the homotopy groups of each $R_{s} X^{(n)}$ are finite in each dimension. Thus $\operatorname{Aut}\left(R_{s} X^{(n)}\right)$ is finite for all $s$. Now given $f: R_{\infty} Y \rightarrow R_{\infty} Y$, there is induced $R_{s}(f): R_{s} R_{\infty} Y$ $\rightarrow R_{s} R_{\infty} Y$. However, the natural map $\phi: Y \rightarrow R_{\infty} Y$ induces $R_{s} \phi: R_{s} Y$ $\rightarrow R_{s} R_{\infty} Y$ which is a homotopy equivalence for good spaces $Y$. That is, there is a natural map from $\operatorname{Aut}\left(R_{\infty} X^{(n)}\right)$ to the tower of groups $\operatorname{Aut}\left(R_{s} X^{(n)}\right)$. The finiteness of $\left[S X^{(n)}, R_{s} X^{(n)}\right]$ implies that $\lim _{s}\left[X^{(n)}, R_{s} X^{(n)}\right] \rightarrow\left[X^{(n)}, R_{\infty} X^{(n)}\right]$ is a bijection, by the short exact sequence of [3] or Quillen [7] for the homotopy groups of the inverse limit of a tower of fibrations. Since $R_{s} X^{(n)}$ and $R_{\infty} X^{(n)}$ are $\mathbf{Z} / p$-complete [3], this holds with $X^{(n)}$ replaced by $R_{\infty} X^{(n)}$.

Thus $\operatorname{Aut}\left(R_{\infty} X^{(n)}\right) \rightarrow \lim \operatorname{Aut}\left(R_{s} X^{(n)}\right)$ is monic. Since $R_{\infty} X^{(n)}=$ $\lim _{\leftarrow} R_{s} X^{(n)}$, it is onto. Therefore $\operatorname{Aut}\left(R_{\infty} X^{(n)}\right)$ is compact, since it is the inverse limit of a tower of finite groups, and the maps $q_{n}$ are continuous since they are induced from families of compatible maps between the finite groups. This completes the proof of Corollary II(c), since $\operatorname{Aut}(\hat{Y})=\Pi_{p} \operatorname{Aut}\left(\hat{Y}_{p}\right)$.

III. An example. We first recall a special case of a theorem of Hilton, Mislin and Roitberg [5].

Lemma 3.1. Let $X$ be a simply connected $C W$-complex with $\pi_{*}(X)$ finitely generated over some subring of $\mathbf{Q}$. Then $\operatorname{Aut}\left(X^{(n)}\right) \rightarrow \operatorname{Aut}\left(X_{0}^{(n)}\right)$ has finite kernel $K_{n}$ for all $n>0$.

Since $\lim ^{1}$ of a tower of finite groups is the one point set, the tailend of the six term exact sequence associated to the short exact sequence of towers of groups [3],

$$
\{1\} \rightarrow\left\{K_{n}\right\} \rightarrow\left\{\operatorname{Aut}\left(X^{(n)}\right)\right\} \rightarrow\{\operatorname{Im}(n)\} \rightarrow\{1\},
$$

degenerates to $* \rightarrow \lim ^{1} \operatorname{Aut}\left(X^{(n)}\right) \rightarrow^{\psi} \lim ^{1} \operatorname{Im}(n) \rightarrow *$, an exact sequence of pointed sets. While this proves that $\psi$ is surjective, it only states that the basepoint is taken to the basepoint by $\psi$. It remains then to show that if 
$\psi(\bar{\beta}) \sim \psi(\bar{\alpha})$, then actually $\bar{\beta} \sim \bar{\alpha}$ in $\lim ^{1} \operatorname{Aut}\left(X^{(n)}\right)$. By the next lemma, this is a general fact about certain short exact sequences of towers of groups.

LEMMA 3.2. If $\{1\} \rightarrow\left\{G_{n}^{\prime}\right\} \rightarrow\left\{G_{n}\right\} \rightarrow^{\psi}\left\{G_{n}^{\prime \prime}\right\} \rightarrow\{1\}$ is an exact sequence of towers of groups, then if $\left\{G_{n}^{\prime}\right\}$ is Mittag-Leffler, $\lim ^{1}(\psi)$ is a bijection of pointed sets.

Proof. If $\bar{\alpha}, \bar{\beta}$ are such that $\psi(\bar{\alpha}) \sim \psi(\bar{\beta})$ in $\lim _{\leftarrow}^{1} G_{n}^{\prime \prime}$, then $\alpha_{n}=\left(\beta_{n} x_{n}\right)$ for some choice of $x_{n}$ in $G_{n}^{\prime}$. Therefore it remains to show only that for each choice of $x_{n},\left(\alpha_{n} x_{n}\right) \sim\left(\alpha_{n}\right)$. The problem is to inductively choose $\gamma_{n+1}$ such that $\left(\gamma_{n+1}\right)_{n}=\left(\alpha_{n}^{-1} \gamma_{n} \alpha_{n}\right) x_{n}$. If $\left\{G_{n}^{\prime}\right\}$ is a tower of epimorphisms, this is easy. But since $\left\{G_{n}^{\prime}\right\}$ is Mittag-Leffler, we can assume up to proisomorphism of towers of groups that $\forall k>0$,

$$
\text { image } \left.G_{n+k}^{\prime} \rightarrow G_{n}^{\prime}\right\}=\left\{\text { image } G_{n+1}^{\prime} \rightarrow G_{n}\right\}=(\text { definition })\left\{G_{n}^{(1)^{\prime}}\right\} \text {. }
$$

Thus we have a ladder:

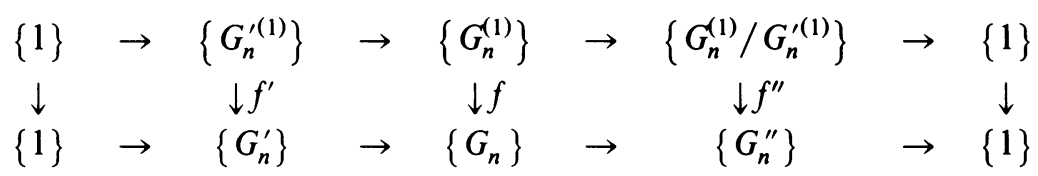

Since $f^{\prime}$ and $f$ are proisomorphisms, $f^{\prime \prime}$ is also. Observe that $\left\{G_{n}^{(1)}\right\}$ is a tower of epimorphisms. Since proisomorphisms induce bijections on $\lim ^{1}$, this concludes the proof.

The proof of Proposition III is the combination of Lemmas 3.1 and 3.2. For the example, we need a space $Y$ with properties similar to those of $\Omega S^{3}$.

Lemma 3.3. There exists a finite type $C W$-space $Y$ such that (a) $Y_{0}$ $\simeq K(\mathbf{Q}, 2)$, (b) $H^{2}(Y, \mathbf{Z})=\mathbf{Z}$ with generator $x_{2}$ such that $x_{2}^{n}$ is divisible by $n^{n}$, and (c) for each prime $p$ and any $b$ in $\mathbf{Z}_{p}$, there is $f_{b}: Y_{p} \rightarrow Y_{p}$ such that $f_{b}^{*} x_{2}=b x_{2}$.

Proof. Construct the Postnikov approximations to $Y$ inductively: $Y^{(2)}$ $=K(\mathbf{Z}, 2)$, and $Y^{(2 n)}$ is induced from $Y^{(2 n-2)}$ by killing the $\mathbf{Z} / n^{n} \mathbf{Z}$ reduction of the image of $x_{2}^{n}$ of $H^{2 n}(K(\mathbf{Z}, 2), \mathbf{Z})$ in $H^{2 n}\left(Y^{(2 n-2)}, \mathbf{Z}\right)$. Thus (b) is immediate and (c) follows from the homogeneity of the $k$-invariants of $Y$.

EXAMPLE 3.4. Let $X=K(\mathbf{Z}, 2) \times Y$, for $Y$ as 3.3. Then for each prime $p$, there exists a sequence of positive integers $v(n)$ such that $v(n) \rightarrow \infty$ as $n \rightarrow \infty$ and such that there is a one-to-one correspondence between $\operatorname{SNT}\left(X_{p}\right)$ and the quotient of the set of $p$-adic integers of the form $\Sigma_{1}^{\infty} b_{n} p^{v(n)}$ under the equivalence relation that $\sum b_{n}^{\prime} p^{(n)} \sim \sum b_{n} p^{v(n)}$ if and only if there exists a $p$-local integer $B$ and a $p$-local unit $A$ such that $A \sum b_{n} p^{v(n)}+\sum b_{n}^{\prime} p^{v(n)}=B$. In particular, $W_{p}$ in $S N T\left(X_{p}\right)$ is homotopy equivalent to $X_{p}$ if and only if the corresponding $p$-adic integer is actually a $p$-local integer. It also follows that $\operatorname{SNT}\left(X_{p}\right)$ is uncountable for each $p$. We conjecture that this example is valid if $Y$ is replaced with $\Omega S^{3}$.

Proof. By Proposition III, it suffices to consider the tower of groups $H^{2}\left(\operatorname{Aut}\left(X_{p}^{(2 n)}\right), \mathbf{Q}\right)$, since this is isomorphic to the tower of images of the automorphism groups in the automorphism groups of the rationalization of the $X_{p}^{(2 n)}$. Consider the "components" of $f: X_{p}^{(2 n)} \rightarrow X_{p}^{(2 n)}: f_{11}: K\left(\mathbf{Z}_{p}, 2\right)$ 
$\rightarrow K\left(\mathbf{Z}_{p}, 2\right), f_{12}: K\left(\mathbf{Z}_{p}, 2\right) \rightarrow Y_{p}^{(2 n)}, f_{21}: Y_{p}^{(2 n)} \rightarrow K\left(\mathbf{Z}_{p}, 2\right)$ and $f_{22}: Y^{(2 n)}$ $\rightarrow Y_{p}^{(2 n)}$. Now $f_{11}, f_{21}$, and $f_{22}$ can induce maps of degree any $p$-local integer on $H^{2}\left(X_{p}^{(2 n)}, \mathbf{Q}\right)$, but the effect of $f_{12}$ is more constrained.

LemMA 3.5. Let $g: \mathbf{C P}(n) \rightarrow Y_{p}$. Then image $H^{2}\left(g, Z_{p}\right)$ is divisible by at least $p^{v(n)}$, with $\max _{j \leqslant n} \nu_{p}(j) \leqslant v(n)$, where $\nu_{p}(j)$ is the exponent of the largest power of $p$ dividing $j$.

Proof. This follows immediately from the structure of the cohomology algebras of $Y$ and $\mathbf{C P}(n)$, using 3.3(b). An upper bound for $v(n)$ can be given in terms of the $p$-primary torsion of the first $2 n$ homotopy groups of $Y$. Any multiple of $p^{v(n)}$ can then be obtained as the degree of $f_{12}$, if $v(n)$ is taken to be the minimum.

With this characterization of $G_{n}=H^{2}\left(\operatorname{Aut}\left(X_{p}^{(2 n)}, \mathbf{Q}\right)\right.$, it is easy to show that each $\left(\alpha_{n}\right) \in \Pi_{n} G_{n}$ is equivalent to

$$
\left\{\left(\begin{array}{cc}
1 & b_{n}^{\prime} p^{v(n)} \\
0 & 1
\end{array}\right)\right\}
$$

for some choice of $\left(b_{n}^{\prime}\right)$. If

$$
\left\{\left(\begin{array}{ll}
a_{n} & r_{n} \\
c_{n} & d_{n}
\end{array}\right)\right\}
$$

has the property that

$$
\left(\begin{array}{ll}
a_{n+1} & r_{n+1} \\
c_{n+1} & d_{n+1}
\end{array}\right)\left(\begin{array}{cc}
1 & s_{n} \\
0 & 1
\end{array}\right)=\left(\begin{array}{cc}
1 & t_{n} \\
0 & 1
\end{array}\right)\left(\begin{array}{ll}
a_{n} & r_{n} \\
c_{n} & d_{n}
\end{array}\right)
$$

for all $n>0$ and the $r_{n}, s_{n}$ and $t_{n}$ satisfy the $p$-divisibility requirements of 3.5 , then by recursion, we have that $r_{1}=d_{1} \sum_{2}^{\infty} t_{n}-a_{1} \sum_{2}^{\infty} s_{n}$ is a valid equation in $\hat{\mathbf{Z}}_{p}$. Conversely, if there exist $p$-local integers $a_{1}, d_{1}$, and $r_{1}$ such that such an expression holds, one can solve for $a_{n}, r_{n}, c_{n}$ and $d_{n}$ and show that

$$
\left\{\left(\begin{array}{cc}
1 & t_{n} \\
0 & 1
\end{array}\right)\right\} \sim\left\{\left(\begin{array}{cc}
1 & s_{n} \\
0 & 1
\end{array}\right)\right\}
$$

in $\lim ^{1} G_{n}$. We remark that the same analysis for $\hat{X}_{p}$ shows that $\operatorname{SNT}\left(\hat{X}_{p}\right)$ $=\left\{\hat{X}_{p}\right\}$ without falling back on Corollary II.

\section{REFERENCES}

1. J. F. Adams, An example in homotopy theory, Proc. Cambridge Philos. Soc. 53 (1957), 922-923. MR 19, 975.

2. V. Belfi and C. Wilkerson, Some examples in the theory of p-completions, Indiana Univ. Math. J. (to appear).

3. A. K. Bousfield and D. M. Kan, Homotopy limits, completions and localizations, Lecture Notes in Math., vol. 304, Springer-Verlag, Berlin and New York, 1972.

4. B. I. Gray, Spaces of the same n-type for all $n$, Topology 5 (1966), 241-243. MR 33 \#4929.

5. P. J. Hilton, Guido Mislin and Joseph Roitberg, Homotopical localization, Proc. London Math. Soc. (3) 26 (1973), 693-706. MR 48 \# 5063.

6. J. P. May, Simplicial objects in algebraic topology, Van Nostrand, Princeton, N. J., 1967. MR 36 \# 5942. 
7. D. G. Quillen, Rational homotopy theory, Ann. of Math. (2) 90 (1969), 205-295. MR 41 \#2678.

8. D. Sullivan, Geometric topology. Part I: Localization, periodicity, and Galois symmetry, M.I.T. Mimeographed notes, Cambridge, 1970; revised, 1971.

9. , Rational homotopy theory (unpublished).

10. C. Wilkerson, Applications of minimal simplicial groups, Topology 15 (1976), 111-130.

Department of Mathematics, University of Pennsylvania, Philadelphia, Pennsylvania 19174 